\title{
Prediction and Migration of Surface-related Resonant Multiples
}

Bowen Guo*, Gerard Schuster, King Abdullah University of Science and Technology, and Yunsong Huang, CGG

\section{SUMMARY}

Surface-related resonant multiples can be migrated to achieve better resolution than migrating primary reflections. We now derive the formula for migrating surface-related resonant multiples, and show its super-resolution characteristics. Moreover, a method is proposed to predict surface-related resonant multiples with zero-offset primary reflections. The prediction can be used to indentify and extract the true resonant multiple from other events. Both synthetic and field data are used to validate this prediction.

\section{INTRODUCTION}

A first-order surface-related resonant multiple is a zero-offset multiple which enjoys two round trips between the free-surface and the common reflector. As shown in Figure 1, the raypaths are coincident with one another. Resonant multiples can be migrated to achieve better resolution than migrating primary reflections. In fact, the imaging resolution can be better than half of the wavelength so that this is considered super-resolution imaging (Schuster and Huang, 2014).

This report firstly derives the migration operator for surfacerelated resonant multiples, and explains why this operator shows super-resolution characteristics. Then, we present the theory of using zero-offset ( $\mathrm{ZO}$ ) primary reflections to predict resonant multiples. This data-based prediction is used to identify and to extract the true resonant multiples in the data. This extraction is required before migration, because the resonant multiples are entangled with other multiple and primary events. Migrating non-resonant multiple events with the resonant multiple migration operator will generate artifacts in the migration image.

After discussing the theory section, synthetic and field data results are shown, and the conclusions are drawn in the last section. function for a source at $\mathbf{g}$ and receiver at $\mathbf{x}$ in a heterogenous medium that honors the 1-way path $\overline{\mathbf{x g}}$ in Figure 1; and $G(\mathbf{x} \mid \mathbf{x})^{M}$ is the 1st-order multiple Green's function that honors the two-way $\overline{\mathbf{x g}^{\prime} \mathbf{x}}$ path in Figure 1 for a source at $\mathbf{x}$ and receiver at $\mathbf{x}$. The recorded 1 st-order resonant data are represented by $d(\mathbf{g})$ for a source and receiver at $\mathbf{g}$ for upcoming pressure waves just below the free surface. Here, $m(\mathbf{x})$ represents the squared reflectivity at the location $\mathbf{x}$ modulated by a -1 amplitude associated with the reflection from the free surface. The reflectivity at $\mathbf{x}$ is squared because the resonant multiple bounces twice from the reflector at $\mathbf{x}$.

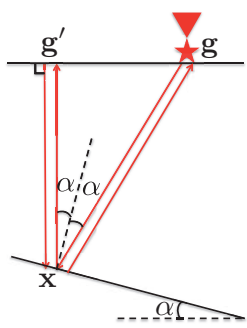

Figure 1: Raypath of a 1st-order resonant multiple in a homogeneous medium with a dipping interface. Star and triangle represent the source and receiver, respectively. The red arrows represent the ray which starts from the source location $\mathbf{g}$, hits $\mathbf{x}$ at the dipping interface, bounces vertically up and hits $\mathbf{g}^{\prime}$ at the free surface boundary. Then the ray repeats its path by going vertically down, reflecting again at $\mathbf{x}$ and is finally recorded by the receiver at $\mathbf{g}$.

The migration formula is the adjoint of equation 1 applied to the data:

$m(\mathbf{x})^{m i g}=\int \omega^{4} \int_{B}\left\{\left[G(\mathbf{g} \mid \mathbf{x})^{D}\right]^{2} G(\mathbf{x} \mid \mathbf{x})^{M}\right\}^{*} d(\mathbf{g}) d x_{g}^{2} d \omega$,

where the integration is over the $2 \mathrm{D}$ recording survey (with locations denoted by the set of points in $B$ ) just below the free surface. Here, $m(\mathbf{x})^{m i g}$ is the migration image of the resonant multiples.

Under high frequency asymptotic assumption (Bleistein et al., THEORY: MIGRATION OPERATOR OF RESONANT MULTIPLES

The Lippmann-Schwinger equations says that the forward modeling formula for a 1st-order resonant multiple from the free surface (Raypath is shown in Figure 1.) can be approximated by

$$
d(\mathbf{g})=\int \omega^{4} \int_{V}\left[G(\mathbf{g} \mid \mathbf{x})^{D}\right]^{2} G(\mathbf{x} \mid \mathbf{x})^{M} m(\mathbf{x}) d x^{3} d \omega,
$$

where $V$ is the set of points in model space and for convenience we assume a wide band source so the wavelet spectrum $W(\omega)=1$. The term $G(\mathbf{x} \mid \mathbf{g})^{D}$ is the direct wave Green's
2001), $G(\mathbf{x} \mid \mathbf{x})^{M}=A(\mathbf{x}, \mathbf{x})^{M} e^{i 2 \omega \tau_{\mathbf{x x}}^{\min }}, G(\mathbf{x} \mid \mathbf{g})^{D}=A(\mathbf{g}, \mathbf{x}) e^{i \omega \tau_{\mathbf{x g}}}$, and $d(\mathbf{g})=A(\mathbf{g}) e^{i \omega \tau_{\mathbf{g}}} . \quad \tau_{\mathbf{x x}}^{\min }$ is the shortest traveltime of the ray $\overline{\mathbf{x g}^{\prime} \mathbf{x}}$, which in practice can be calculated by a plane-wave eikonal solver. $\tau_{\mathbf{x g}}$ is the traveltime of the direct arrival from $\mathbf{g}$ to $\mathbf{x} . \tau_{\mathbf{g}}$ is the traveltime of the 1st-order resonant multiples. Neglecting the amplitude parts, the kernel in equation 2 can be approximated by

$$
\left.\left\{G(\mathbf{g} \mid \mathbf{x})^{D}\right]^{2} G(\mathbf{x} \mid \mathbf{x})^{M}\right\}^{*} d(\mathbf{g}) \approx e^{i \omega\left(\tau_{\mathrm{g}}-2\left(\tau_{\mathbf{x x}}^{\min }+\tau_{\mathbf{x g}}\right)\right)} .
$$

For a fixed $\tau_{\mathbf{g}}, \tau_{\mathbf{g}}-2\left(\tau_{\mathbf{x x}}^{\min }+\tau_{\mathbf{x g}}\right)=0$ defines an isochron in the model space, which in a homogeneous medium can be analytically formulated as

$$
z=\frac{t_{m} v}{4}-\frac{\left(x-x_{\mathbf{g}}\right)^{2}}{t_{m} v}
$$




\section{Surface-related Resonant Multiples}

where $v$ is the velocity of the homogeneous medium, and $z$ and $x$ are the vertical and horizontal component of $\mathbf{x}$, respectively. This isochron is shown as the red curve in Figure 2a. If the traveltime of a resonant multiple varies from $t_{\mathrm{g}}-T_{0} / 2$ to $t_{\mathrm{g}}+T_{0} / 2$, where $T_{0}$ is the dominant period of the resonant multiple wavelet, the isochron changes correspondingly from the inner to the outer dashed blue line in Figure 2a. Between the two lines is the migration response of a single resonant multiple wavelet, which is defined as the migration operator of resonant multiples. Samilar analysis can be applied to the $\mathrm{ZO}$ primary reflection case, and its migration operator is shown in Figure $2 \mathrm{~b}$. The comparison between Figures $2 \mathrm{a}$ and $2 \mathrm{~b}$ shows the resonant multiple operator is skinnier and so has a better resolution than the $\mathrm{ZO}$ primary reflection operator. For example, for the image point directly below the source/receiver position $\left(x=x_{g}\right)$, the thickness of the resonant multiple operator is a quarter of the wavelength, which is half compared to the primary reflection operator. In other words, resonant multiple migration shows subwavelength (also called supperresolution) characteristics.

(a) Migration Operator of Resonant Multiples

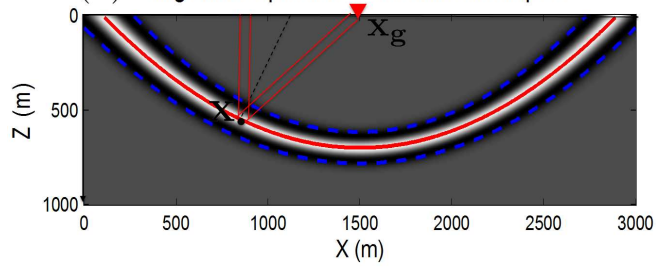

(b) Migration Operator of primary reflections

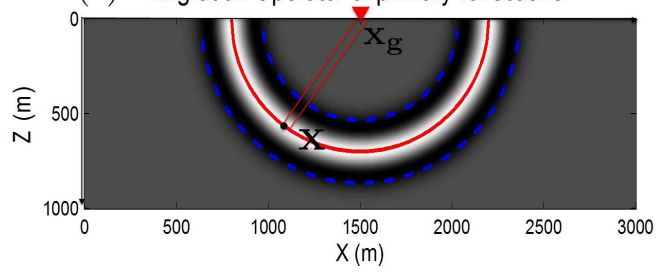

Figure 2: Single-trace migration operators for (a) resonant multiples, and (b) primary reflections.

A synthetic experiment is designed to illustrate super-resolution imaging achieved by migrating resonant multiples. As shown in Figure 3, 16 point scatterers are placed in the model. In Figure $3 \mathrm{a}$, diffractions of the point scatters are generated by Kirchhoff modeling, and then migrated by Kirchhoff migration to obtain the migration image. In Figure $3 b$, the resonant multiples, generated by equation 1 , are migrated by equation 2. The comparison between Figures $3 \mathrm{a}$ and $3 \mathrm{~b}$ shows that the migration image of resonant multiples has finer resolution. Especially, the top four point scatterers which are a quarter wavelength apart are distinguishable in the resonant multiple migration image.
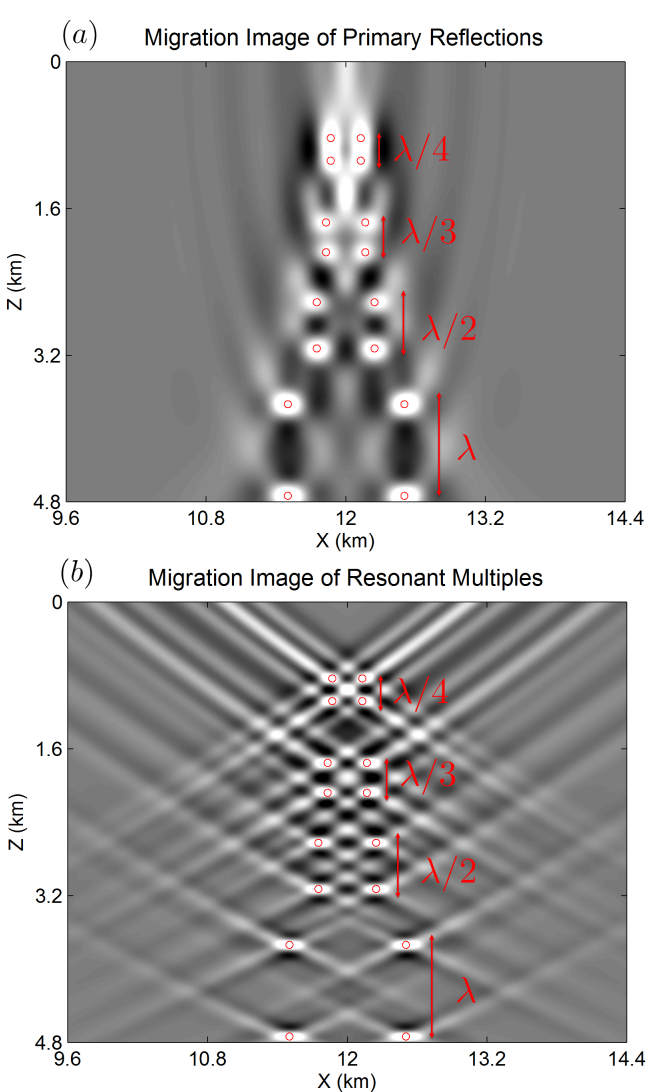

Figure 3: Migration images of point scatters by migrating (a) primary reflections and (b) resonant multiples. The red dots are the positions of the point scatterers. $\lambda$ represents the wavelength corresponding to the data wavelet.

\section{THEORY: PREDICTION OF RESONANT MULTIPLES WITH PRIMARY REFLECTIONS}

Separation of the resonant multiples is needed before migration. We use ZO primary reflections to predict and so extract resonant multiples. Resonant multiple $d_{m}\left(t_{m}, \mathbf{g}_{\mathbf{m}}\right)$ recorded at time $t_{m}$ at $\mathbf{g}_{\mathbf{m}}$ can be predicted by

$$
\text { where } \begin{gathered}
d_{m}\left(t_{m}, \mathbf{g}_{\mathbf{m}}\right)=\sum d_{p}\left(t_{p}, \mathbf{g}_{\mathbf{p}}\right), \\
t_{m}=\tau\left(\mathbf{g}_{\mathbf{m}} ; t_{p}, \mathbf{g}_{\mathbf{p}}\right),
\end{gathered}
$$

and $d_{p}\left(t_{p}, \mathbf{g}_{\mathbf{p}}\right)$ is a $\mathrm{ZO}$ primary reflection signal recorded at time $t_{p}$ at $\mathbf{g}_{\mathbf{p}}$. The summation is along an isochon represented by $t_{m}=\tau\left(\mathbf{g}_{\mathbf{m}} ; t_{p}, \mathbf{g}_{\mathbf{p}}\right)$ in the data space. The construction of this isochon in equation 5 , that is similar to the migration to zerooffset operation for primaries (Tygel et al., 1998; Schleicher and Bagaini, 2004) can be summerized as a two-step process shown in Figure 4a.

- Step 1: In a ZO primary reflection data set, for each time sample $\left(t_{p}, \mathbf{g}_{\mathbf{p}}\right)$, we build an isochron in the model space. The next step is to use this model space isochron to build the isochron in the data space.

- Step 2: Use the model space isochron as a reflector to forward model resonant multiples. That is, given a 


\section{Surface-related Resonant Multiples}

point at the model space isochron $\mathbf{x}$, calculate the first ray which starts from $\mathbf{x}$ and reflects normally from the free-surface boundary. Based on this ray and the local dip of the isochon at $\mathbf{x}$, find the second ray which obeys the reflection law. The intersection between the second ray and the free-surface boundary is $\mathbf{g}_{\mathbf{m}}$, and twice the total length of the two rays gives the resonant multiple travel time $t_{m}$. Repeat this process for all the $\mathbf{x}$ along the model space isochron so that the data space isochron is determined.

In Figure $4 b$, the red curve is the isochron in the resonant multiple data space, associated with the $\mathrm{ZO}$ primary reflection signal represented by the red dot. The result of summing the isochrons associated with the $\mathrm{ZO}$ primary reflections is shown in Figure 4c, and the envelop of these of isochrons matches the trajectory of the true resonant multiples.

Figure 5 shows the comparison between the true and predicted resonant multiples based on equation 5 . The true multiples are a part of the data generated by finite difference (FD) method with a free-surface boundary condition based on a two-layer velocity model with a dipping interface. The predicted and the resonant multiples match quite well. Notice the wavelets of the predicted resonant multiples are streched compared to the true multiple wavelets. This strech is because of the property of the isochron $t_{m}=\tau\left(\mathbf{g}_{\mathbf{m}} ; t_{p}, \mathbf{g}_{\mathbf{p}}\right)$, that is when $t_{p}$ is perturbed by $T_{0}$, the corresponding perturbation in $t_{m}$ is longer than $T_{0}$.

\section{NUMERICAL RESULTS}

Figure 6a shows a common offset gather (142 m offset) of a marine data set. The events above the blue dashed line are mostly primary reflections, and the events below contain resonant multiple events. A moveout correction and stacking are applied to the primary reflections and to the multiples separately based on their moveout formula. Figure $6 \mathrm{~b}$ shows the post-stack marine data. Compared with Figure $6 \mathrm{a}$, the signalto-noise ratio in Figure $6 \mathrm{~b}$ is strengthed. We then use the poststack primary reflections in Figure $7 \mathrm{~b}$ to predict resonant multiples based on equation 5 . The prediction results are shown in Figure 7a. This prediction can be used as a reference to identify and to window the resonant multiples in Figure 7b. Also, the predicted resonant multiples say that the third event between the two red dashed lines (indicated by the blue arrow) in Figure $7 \mathrm{~b}$ is not a resonant multiple.

Figures 8 and 9 show the prediction and migration of resonant multiples in another near-offset (108 m offset) marine data set. The near-offset primaries are used to predict resonant multiples (Figure 8b), and the prediction says that the event indicated by the blue arrow in Figure $8 \mathrm{a}$ is a non-resonant multiple event. Migration image of the resonant multiples is shown in Figure 9b. Compared with the post-stack primary image (Figure 9a), resonant multiple image has better resolution.
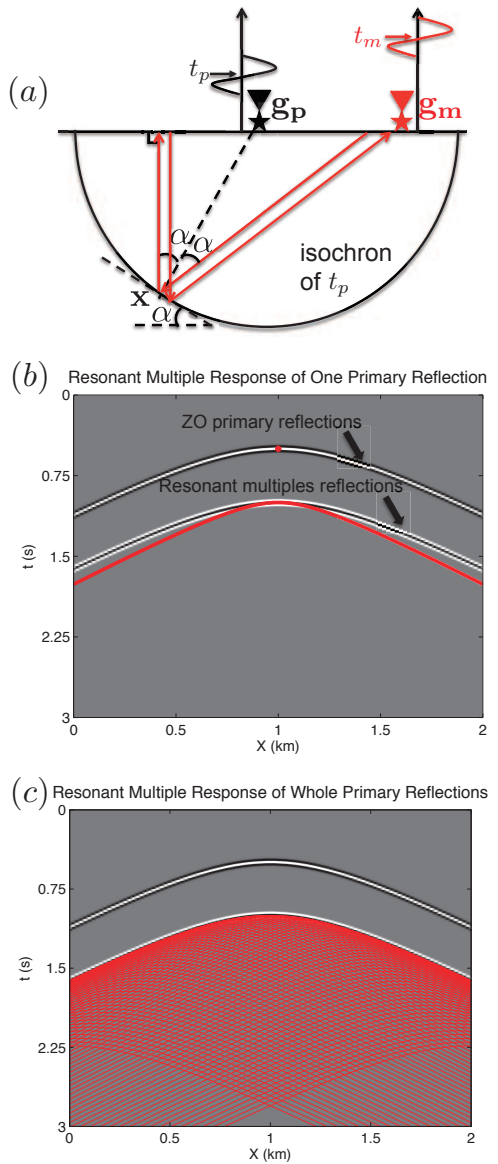

Figure 4: Illustration of predicting resonant multiples with ZO primary reflections. (a) Transformation from the ZO primary reflection signal recorded at $t_{p}$ by a trace at $\mathbf{g}_{\mathbf{p}}$ to the resonant multiple signal recorded at $t_{m}$ by a trace at $\mathbf{g}_{\mathbf{m}}$. (b) The isochron of the resonant multiples (red curve) associated with the ZO primary reflection signal recorded at $t_{p}$ at $\mathbf{g}_{\mathbf{p}}$ (red dot). (c) A group of isochrones in the resonant multiples (red curves) associated with all of the ZO primary reflections. The envelop of the red curves matches the trajectory of the true resonant multiples.

\section{CONCLUSIONS}

We derive the migration formula for surface-related resonant multiples. This migration operator has better resolution than $\mathrm{ZO}$ primary reflections migration operator. We also show how $\mathrm{ZO}$ primary reflections can be used to predict 1st-order resonant multiples. The prediction of resonant multiples can be used to indentify and window the resonant multiples from other events, as shown by a marine seismic data set. The result of another marine data set shows the subwavelength characteristics of imaging resonant multiples. 


\section{Surface-related Resonant Multiples}

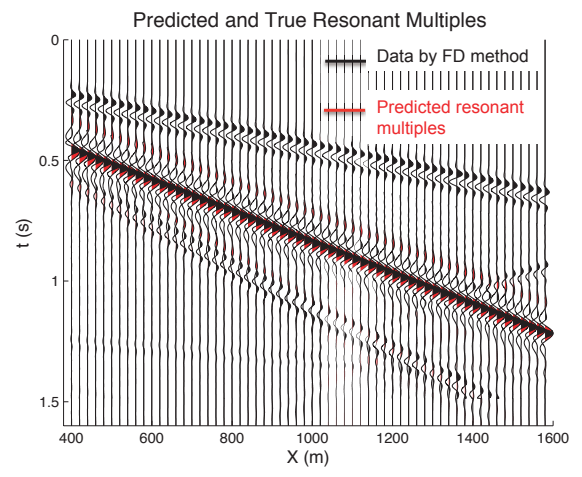

Figure 5: Comparison of the predicted and the true resonant multiples in the case of a dipping interface. The black wiggles represent the true resonant multiples, and among them the second event is the 1st-order surface-related resonant multiple (amplitude amplified). The red wiggles represent the predicted resonant multiples.
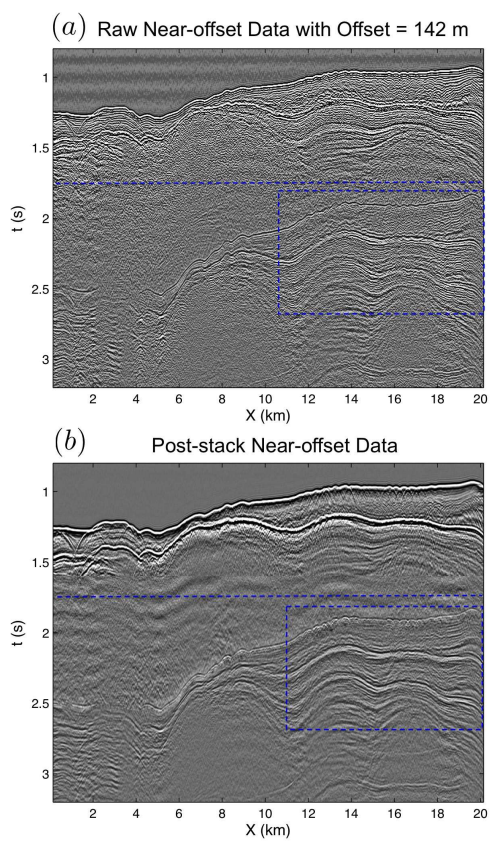

Figure 6: (a) A pre-stack near offset marine data set. (b) Poststack data. In panels a and b, events above the blue dashed line are mostly primary reflections, and the events below contain resonant multiples. The blue dashed square sections highlight the better signal-to-noise ratio of post-stack data.
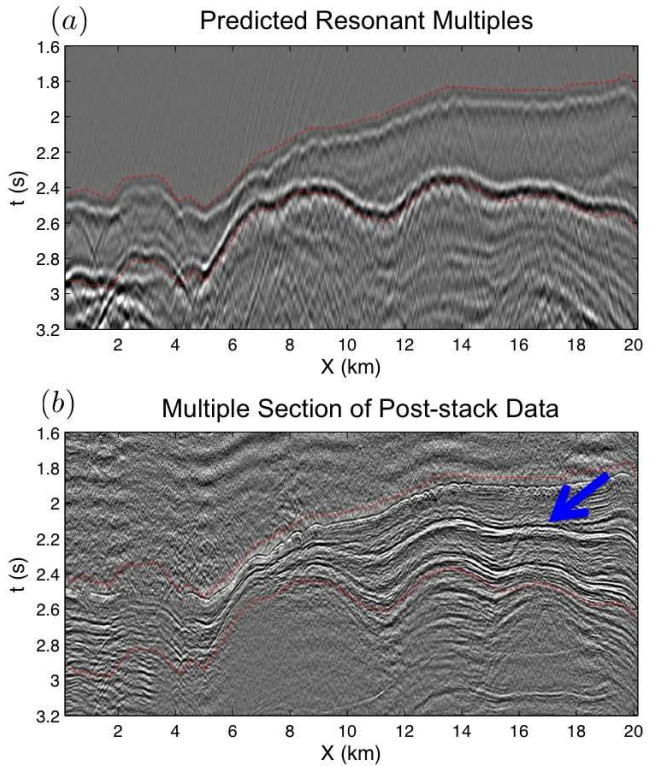

Figure 7: Comparison between (a) the predicted resonant multiples and (b) the post-stack multiple events. The red dashed lines show that the predicted resonant multiples can be used to window resonant multiples. The blue arrow indicates a nonresonant multiple event.
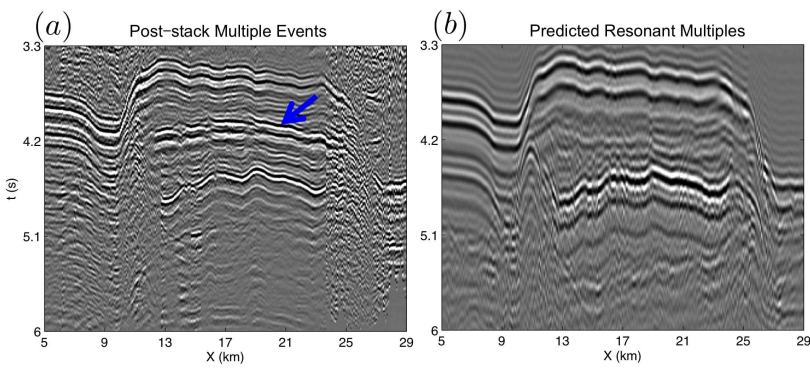

Figure 8: Comparison between (a) post-stack multiple events and (b) predicted resonant multiples from $\mathrm{ZO}$ primaries. The prediction in panel $\mathrm{b}$ indicates a non-resonant multiple event (blue arrow) in panel a.
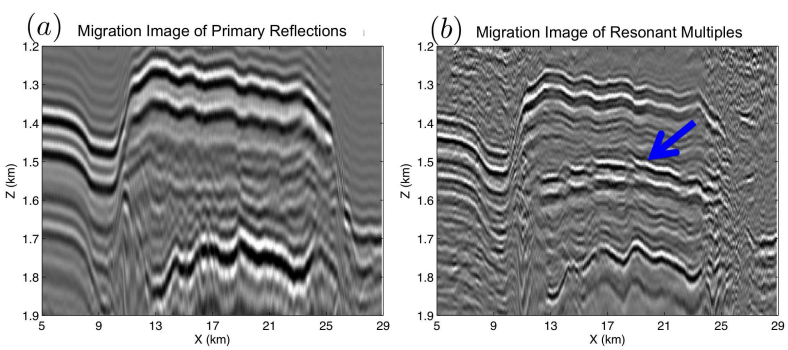

Figure 9: Comparison between (a) primary reflections migration image and (b) resonant multiples migration image. Panel b shows better resolution than panel a. The blue arrow indicates migration artifacts caused by non-resonant multiple events. 


\section{EDITED REFERENCES}

Note: This reference list is a copyedited version of the reference list submitted by the author. Reference lists for the 2015 SEG Technical Program Expanded Abstracts have been copyedited so that references provided with the online metadata for each paper will achieve a high degree of linking to cited sources that appear on the Web.

\section{REFERENCES}

Bleistein, N., J. Cohen, and W. J. Stockwell, 2001, Mathematics of multidimensional seismic imaging, migration, and inversion: Springer Science \& Business Media. http://dx.doi.org/10.1007/978-1-4613-0001-4.

Schleicher, J., and C. Bagaini, 2004, Controlling amplitudes in $2.5 \mathrm{~d}$ common-shot migration to zero offset: Geophysics, 69, 1299-1310. http://dx.doi.org/10.1190/1.1801946.

Schuster, G. T., and Y. Huang, 2014, Far-field super-resolution by imaging of resonance: Geophysical Journal International, 199, 1-7.

Tygel, M., J. Schleicher, P. Hubral, and L. T. Santos, 1998, 2.5-d true-amplitude Kirchhoff migration to zero offset in laterally inhomogeneous media: Geophysics, 63, 557-573. 